\title{
Phase Separation and Network Formation in Poly(vinyl methyl ether)/Water Solutions
}

\author{
Yanyin YANG, Fang ZENG, Xiaoli XIE, Zhen TONG, ${ }^{\dagger}$ and Xinxing LIU \\ Research Institute of Materials Science, South China University of Technology, \\ Guangzhou 510640, China
}

(Received October 25, 2000; Accepted January 20, 2001)

\begin{abstract}
Cloud point temperature $T_{\text {clo }}$ and dynamic moduli were measured on aqueous solutions of two poly(vinyl methyl ether) (PVME) samples with molecular weight of $1.16 \times 10^{4}$ and $2.57 \times 10^{4}$, respectively and $M_{\mathrm{w}} / M_{\mathrm{n}}=1.84$ for both samples. The $T_{\text {clo }}$ at which the solution becomes turbid is at around $32.5^{\circ} \mathrm{C}$ without obvious molecular weight dependence due to their broad molecular weight distribution. The frequency $\omega$ dependence of the storage modulus $G^{\prime}$ and loss modulus $G$ " observed within linear viscoelasticity region indicates that the PVME/water solution exhibits homogeneous fluid behavior at $25^{\circ} \mathrm{C}$ below $T_{\text {clo }}$, while shows a plateau zone for $G^{\prime}$ at $34^{\circ} \mathrm{C}$ above $T_{\text {clo }}$, suggesting the appearance of network structure at this temperature. A sharp increase in $G^{\prime}$ and $G^{\prime \prime}$ was observed at about $32.5^{\circ} \mathrm{C}$ with increasing temperature of a PVME/water solution, indicating that at this temperature the network begin to form. These results imply that the network structure is formed accompanying the phase separation in PVME/water solutions due to the hydrophobic bonding between polymer chains.
\end{abstract}

KEY WORDS Poly(vinyl methyl ether) (PVME) / Network Formation / Hydrophobic Bonding / Phase Separation/

The gelation is a universal phenomenon in solutions of both biopolymers and synthetic macromolecules due to the formation of crosslinks among the molecular chains. According to the nature of the crosslinks, the gelation can be classified into two categories: chemical and physical. In the latter, the gelation process is usually reversible and the gel is characterized by the appearance of pronounced plateau in its storage modulus $G^{\prime}$ and much smaller loss modulus $G^{\prime \prime}$ in the same frequency region. ${ }^{1}$ The predominant interaction, which induces the molecule aggregation, is of physical, e.g., micellar crystallite, helix formation, hydrogen bonding, ionic coordination, and complex formation. Phase separation caused physical gelation is owing to either the glassy transition in polymer rich phase as found in polystyrene/cyclohexanol $^{2}$ or the partial solubility of block copolymers in their selective solvents. ${ }^{3}$

Recently, we reported a new type of physical network formed by simple hydrophobic bonding in $\operatorname{poly}(N-$ isopropyl acrylamide) (PNIPAm)/water solutions. ${ }^{4-8}$ PNIPAm, containing both hydrophilic amide groups and hydrophobic isopropyl groups in its side chains, is an attractive polymer because its water solution undergoes phase separation upon heating exhibiting a lower critical solution temperature (LCST) at about $32^{\circ} \mathrm{C} .{ }^{9}$ In addition, the chemically crosslinked PNIPAm hydrogel undergoes a sudden volume shrinkage in water when heated above $33.2{ }^{\circ} \mathrm{C} .{ }^{10}$ Dynamic viscoelasticity measurements indicate the formation of thermoreversible network structures in aqueous solutions of PNIPAm with viscosity-averaged molecular weight of $2.1 \times 10^{6}$ even at concentration as low as $1.22 \mathrm{wt} \%$ during the phase separation. ${ }^{4} \mathrm{NMR}$ study on this system encompassing the phase separation reveals that during the phase separation the ordered water molecules surrounding hydrophobic $N$-isopropyl groups are dissociated from the hydrated shell and the hydrophobic bonding is formed among these groups. ${ }^{11}$ When the solution is concentrated enough, the hydrophobic bonding can be formed among the $N$-isopropyl groups attaching to different PNIPAm chains, which causes a network structure in the PNI$\mathrm{PAm} /$ water solution during the phase separation.

However, when we are stating that the hydrophobic bonding induces the network structure in PNIPAm/ water solutions, the possible contribution from intermolecular hydrogen bonds between amide groups cannot be clearly separated from the others. To confirm effectively that the hydrophobic bonding does cause the physical gelation during phase separation, a special polymer is required, which undergoes phase separation in water solution induced by hydrophobic bonding without any intermolecular hydrogen bonds. For this purpose, we choose poly(vinyl methyl ether) (PVME) in this work, whose aqueous solution turns into turbid suspension upon heating to around $33^{\circ} \mathrm{C}$ and exhibits $\mathrm{LCST}^{12}$ and molecular chains cannot form any inter- or intra-molecular hydrogen bonds. Horne et al. ${ }^{12}$ have reported the phase separation behavior of aqueous PVME solutions and the effect of added salts and alcohols. Schild and Tirrell ${ }^{13}$ found that the cloud point temperature $T_{\text {clo }}$ was independent of molecular weight for two PVME/water solutions measured by microcalorimetry and argued that the origin of phase separation for PVME/water solution was similar to that of the PNIPAm/water system.

In this work, we will reveal experimentally the correlation of phase separation and network formation in PVME/water solutions upon heating.

\section{EXPERIMENTAL}

Samples

Two poly(vinyl ethyl ether) samples (PVME-12 and

\footnotetext{
${ }^{\dagger}$ To whom correspondence should be addressed (Tel and Fax: +86-20-87112886, E-mail: mcztong@scut. edu. cn).
} 
PVME-26) in water solution were purchased from Tokyo Chemical Industry Co., Ltd. (Japan) and Scientific Polymer Products Inc. (USA) respectively. Their molecular weight was determined by measuring the intrinsic viscosity of PVME in methyl ethyl ketone solution at $30^{\circ} \mathrm{C}$ using the Mark-Houwink equation $[\eta]=0.137 M_{\eta}^{0.56}{ }^{14}$ and the molecular weight distribution index $M_{\mathrm{w}} / M_{\mathrm{n}}$ was estimated with a Water-150C GPC at $30^{\circ} \mathrm{C}$ using THF as the elution phase and narrow distributed polystyrene as the standard. The determined molecular weight $M_{\eta}$ for these PVME samples was $1.16 \times 10^{4}$ and $2.57 \times 10^{4}$, referred to as PVME-12 and PVME-26, respectively; their $M_{\mathrm{w}} / M_{\mathrm{n}}$ was 1.84 for both samples. These two original PVME samples in water solution were first freezingdried and their solid contents were determined by weighing. Doubly distilled water was used to dissolve the PVME samples to the required concentration.

\section{Cloud Point Measurement}

Desired amount of PVME sample was added into a cylindrical cell, dissolved with proper amount of water and sealed for cloud point measurement. Cloud point temperature of PVME/water solutions was determined by monitoring the change in solution transmittance with increasing the solution temperature gradually at rate of $2^{\circ} \mathrm{C} \mathrm{h}^{-1}$ as described before. ${ }^{5}$ The solution transmittance was determined according to the intensity of a laser beam passing the solution. The temperature at which the solution transmittance began to suddenly reduce was designated as the cloud point temperature $T_{\text {clo }}$. The cloud point temperature determined was reproducible either by increasing the temperature once more from about 3 degrees below $T_{\text {clo }}$ or by decreasing the temperature from about 3 degrees above $T_{\text {clo }}$.

\section{Viscoelasticity Measurement}

The viscoelasticity was detected by a Rheometrics RFS-II strain-controlled rheometer with a Couette fixture whose cup and bob diameter is $33.96 \mathrm{~mm}$ and 32.00 $\mathrm{mm}$, respectively, with length of $33.10 \mathrm{~mm}$. Temperature was controlled by a computer-programmable circulator with a precision of $\pm 0.1^{\circ} \mathrm{C}$. Four modes of viscoelastic measurements were conducted in the present work. The first is the steady-flow viscosity $\eta$ at $25^{\circ} \mathrm{C}$ as a function of shear rate $\dot{\gamma}$ ranging from $10^{-1}$ to $10^{2} \mathrm{~s}^{-1}$; second the complex modulus $G^{*}$ at $25^{\circ} \mathrm{C}$ and $34^{\circ} \mathrm{C}$ as a function of shear strain $\gamma$ ranging from $10^{-2}$ to $10^{\circ}$ at a fixed angular frequency $\omega$ of $20 \mathrm{rad} \mathrm{s}^{-1}$, third the storage modulus $G^{\prime}$ and loss modulus $G^{\prime \prime}$ as a function of frequency $\omega$ ranging from $10^{-1}$ to $10^{2} \mathrm{rad} \mathrm{s}^{-1}$ at a fixed shear strain $\gamma$ of 0.2 at $25^{\circ} \mathrm{C}$ below the phase separation temperature, and at $34^{\circ} \mathrm{C}$ above the phase separation, respectively and the last the storage modulus $G^{\prime}$ and loss modulus $G^{\prime \prime}$ as a function of temperature raised from $24^{\circ} \mathrm{C}$ to $35^{\circ} \mathrm{C}$ at the rate of $0.1^{\circ} \mathrm{C} \min ^{-1}$.

\section{RESULTS AND DISCUSSION}

\section{Cloud Point Curve}

Figure 1 shows the plots of cloud point temperature $T_{\text {clo }}$ vs. concentration of PVME/water solution for our two PVME samples. No obvious concentration dependence of cloud point temperature can be recognized from

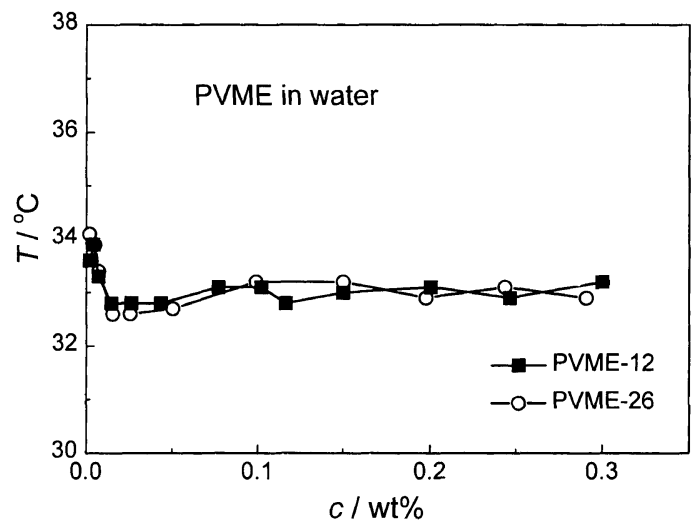

Figure 1. Plots of cloud point temperature $T_{\text {clo }}$ against polymer concentration $c$ for PVME/water solutions.

these two curves, except in dilute region, where $T_{\text {clo }}$ abruptly increases with the decrease of concentration. An important feature of this figure may be that the two cloud point curves almost overlap, implying the independence of cloud point temperature $T_{\text {clo }}$ on the molecular weight of PVME samples. However, we cannot conclude that $T_{\text {clo }}$ of PVME/water solutions is independent of molecular weight and concentration at present, because the molecular weight for these two PVME samples are rather close to each other and furthermore their molecular weight distributions are too broad $\left(M_{\mathrm{w}} / M_{\mathrm{n}}=\right.$ 1.84) to discuss the solution thermodynamic properties strictly. This is apparently a common phenomenon for aqueous solutions of amphiphilic polymers induced by hydrophobic interaction, but the cloud point temperature does substantially depend on the molecular weight of the polymer if its distribution is narrow enough, e.g., poly $(N$-isopropyl acrylamide) (PNIPAm) in water.,7

Compared with the phase separation driven by dissimilarity in the free volume of polymer and solvent, such as polystyrene/cyclohexane solution which separates into two liquid phases at an elevated temperature about $237^{\circ} \mathrm{C}^{15}$ the phase separation of PVME/water solution occurs at a temperature much lower than the boiling point of the solvent, and the concentration dependence of cloud point temperature is not so obvious. Therefore, different mechanism should be considered for the phase separation in PVME/water solutions.

As for the affinity to water, PVME is an amphiphilic polymer, where the alkane backbone and the methyl groups on side chain are hydrophobic while the ether bond is hydrophilic. The oxygen atom in the ether bond can join hydrogen bond with water molecules thus making PVME soluble in water, but hydrogen bond is never formed between repeat units of PVME. The phase separation in PVME/water solutions may be attributed to the hydrophobic bonding formed upon heating. According to Nemethy and Scheraga, ${ }^{16-18}$ at the temperature below $T_{\text {clo }}$, water molecules around the hydrophobic groups form a hydrated sheath with ice-like structure through hydrogen bonds between water molecules themselves to protect the hydrophobic groups to contact each other. When heated to $T_{\text {clo }}$, these hydrogen bonds will be broken; the order structure of the hydrated sheath is destroyed. With releasing water molecules from the hydrated sheath around the hydrophobic moiety of molecu- 


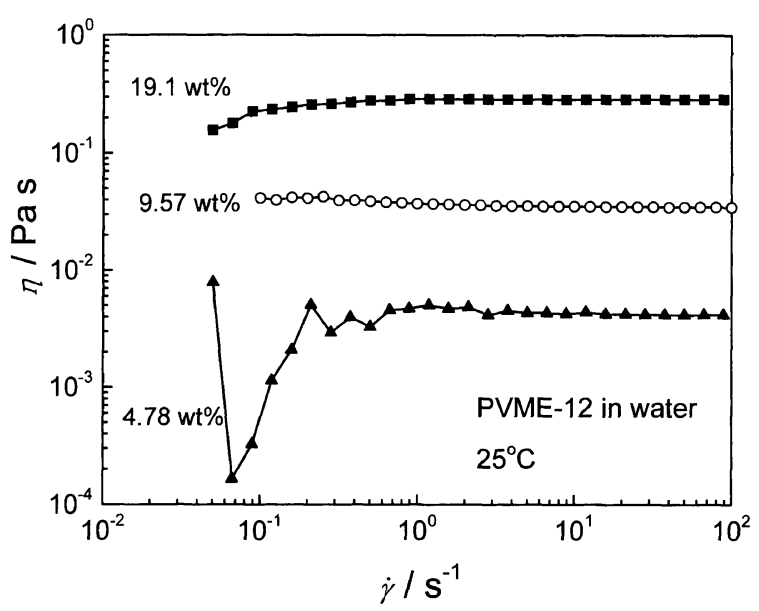

Figure 2. Shearing viscosity $\eta$ of PVME-12/water solution of indicated concentrations at $25^{\circ} \mathrm{C}$ as a function of shear rate $\dot{\gamma}$.

lar chains to the bulk solvent, the hydrophobic groups become closer to each other and finally fall within the van der Waals radius, leading to the formation of hydrophobic bonding. This process has been proved for the phase separation in PNIPAm/water solution accompanying the network formation crosslinked by the hydrophobic bonding among different macromolecular chains. $^{4,11}$ That the network structure appears meanwhile the phase separation occurs will be a powerful evidence for the hydrophobic bonding in PVME/water solution.

\section{Shear Viscosity}

The shear viscosity $\eta$ of PVME-12/water solutions of $4.78,9.57$, and $19.1 \mathrm{wt} \%$ respectively, at $25^{\circ} \mathrm{C}$ is depicted in Figure 2 as a function of shear rate $\dot{\gamma} . \eta$ for all these three solutions is increased with polymer concentration and almost independent of $\dot{\gamma}$. For PVME/water solution of $4.78 \mathrm{wt} \%$ at low shear rate $\left(<0.3 \mathrm{~s}^{-1}\right)$, the data scatters due to the torque sensitivity limitation of the transducer. Thus, below the phase separation temperature, the PVME/water solution behaves like a Newtonian viscous fluid even at polymer concentration as high as 19.1 wt\%. This is owing to the relative low molecular weight of the PVME-12.

\section{Network Formation Accompanying Phase Separation}

The relationship between the complex modulus $G^{*}$ and shear strain $\gamma$ for PVME-12 in water solution of 9.57 and $19.1 \mathrm{wt} \%$ is illustrated in Figure 3 . At $25^{\circ} \mathrm{C}$ below the cloud point temperature, the complex modulus $G^{*}$ does not change with $\gamma$ in the measured strain $\gamma$ range from 0.01 to 1.0 . And at $34^{\circ} \mathrm{C}$ above the cloud point temperature, $G^{*}$ remains unchanged with $\gamma$ until $\gamma$ is higher than 0.2 . There is a common linear viscoelasticity region for PVME/water solution when $\gamma$ is not larger than 0.2 , in spite of below or above the cloud point temperature. The same linear region was also observed for PVME-26 in water at various concentrations, so that we keep the oscillatory strain at 0.2 in the following experiments to produce enough torque as well as to ensure the linear viscoelasticity available.

In order to investigate the structure change in PVME/

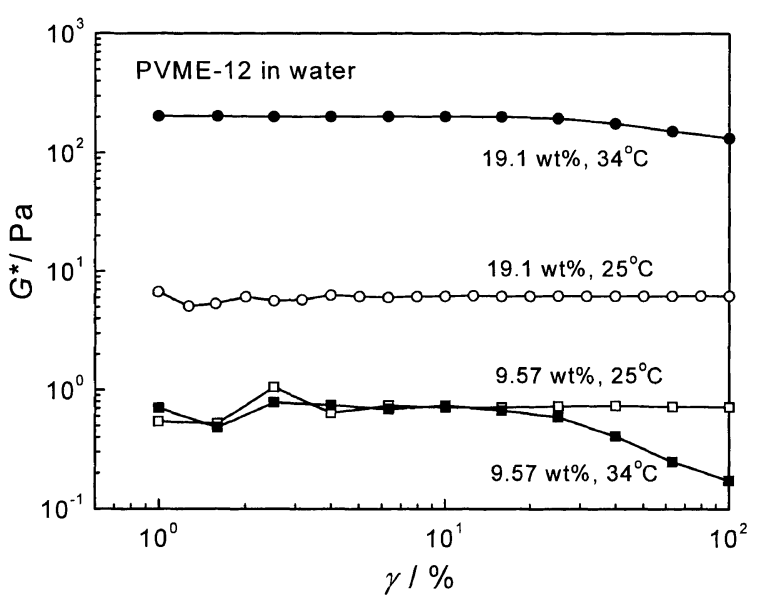

Figure 3. Strain $\gamma$ dependence of complex modulus $G^{*}$ for two PVME-12/water solutions at $25^{\circ} \mathrm{C}$ and $34^{\circ} \mathrm{C}$.

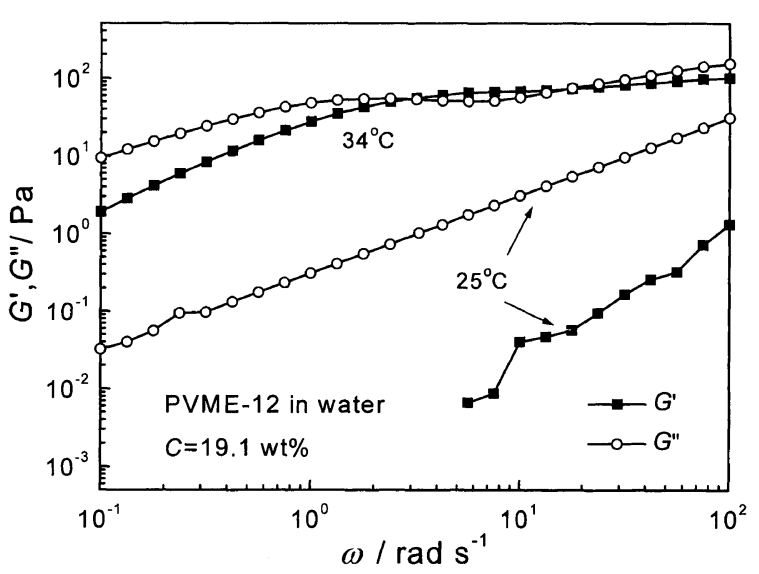

(a)

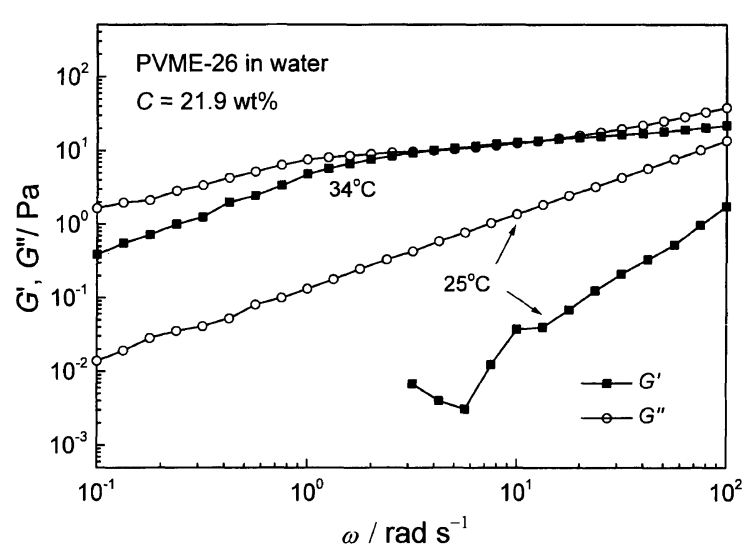

(b)

Figure 4. Plots of dynamic moduli $G^{\prime}$ and $G^{\prime \prime}$ against angular frequency $\omega$ for aqueous solution of PVME-12 (a) and PVME-26 (b) at $25^{\circ} \mathrm{C}$ and $34^{\circ} \mathrm{C}$.

water solution accompanying the phase separation, we measured the dynamic modulus of the solution before phase separation at $25^{\circ} \mathrm{C}$ and after phase separation at $34^{\circ} \mathrm{C}$ and show the results in Figure 4 . In Figure $4 \mathrm{a}$, the storage modulus $G^{\prime}$ and loss modulus $G^{\prime \prime}$ are plotted against the angular frequency $\omega$ for PVME-12/water solution of $19.1 \mathrm{wt} \%$ at $25^{\circ} \mathrm{C}$ and $34^{\circ} \mathrm{C}$, respectively. It can 
be seen that at $25^{\circ} \mathrm{C}$, both $G^{\prime \prime}$ and $G^{\prime}$ increase with increasing frequency, and $G^{\prime \prime}>G^{\prime}$ throughout. Besides, $G^{\prime \prime}$ and $G^{\prime}$ are proportionate to $\omega^{1.0}$ and $\omega^{1.77}$, respectively. Due to rather broad molecular weight distribution of our PVME samples, the theoretically predicted $\omega^{2.0}$ cannot be reached. Also within the tested frequency range, no modulus plateau appears in the storage modulus $G^{\prime}$, indicating that no molecular network is formed at this temperature.

However, the behavior is totally different when temperature is increased to $34^{\circ} \mathrm{C}$ above the $T_{\text {clo }}$. Not only both $G^{\prime}$ and $G^{\prime \prime}$ increase significantly, but also a plateau of $G^{\prime}$ appears within the frequency range of $2.5-10^{2}$ $\operatorname{rad~s}^{-1}$ and $G^{\prime}$ becomes much closer to or even larger than $G$ " over frequencies. This is a typical characteristic for the network formation in the solution. ${ }^{19}$ Although relaxation of the network chains can be still observed at low frequencies due to the weak interaction exerting at the crosslinking joints and low molecular weight of PVME samples used here. Similar phenomena also occur in PVME-26/water solution of $21.9 \mathrm{wt} \%$ as shown in Figure $4 \mathrm{~b}$.

Hydrogen bond cannot be constructed between the repeat units of PVME anyway due to the lack of hydrogen donor in its segment structure. Therefore, the network structure formed in PVME/water solution accompanying phase separation should be merely caused by intermolecular hydrophobic bonding, like that in PNIPAm/ water solution. ${ }^{4,5}$ When the polymer concentration is higher than the overlap concentration, crosslinking will occur among polymer chains, causing the network structure crosslinked by the hydrophobic bonding.

\section{Gelation Process}

As described in Experimental, the PVME/water solution changes from a transparent homogenous fluid into a turbid viscoelastic state during phase separation. The crosslinked network found here is rather weak, so it can still flow, unlike other systems in which the solution becomes a macroscopic viscoelastic solid after sol-gel transition. According to te Nijenhuis's definition of gel, ${ }^{1}$ in which modulus has a plateau within an observable frequency range, the structure formed in PVME/water solutions after phase separation is classified as a gel although it is not strong. We believe that the short relaxation time of the network is due to the weak hydrophobic bonding induced only by the methyl group and backbone of PVME chain. If the hydrophobic moiety is enlarged, the crosslink joints will be enhance, resulting in a longer relaxation as observed from PNIPAm/water system after phase separation. ${ }^{5}$

To trace the gelation process, we increased the temperature of PVME-26 (21.9 wt\%)/water solution from $24^{\circ} \mathrm{C}$ to $35^{\circ} \mathrm{C}$ at a rate of $0.1^{\circ} \mathrm{C} \min ^{-1}$, and recorded the storage modulus $G^{\prime}$ and loss modulus $G^{\prime \prime}$ as a function of temperature as given in Figure 5. It can be seen that when temperature is lower than $29^{\circ} \mathrm{C}, G^{\prime}$ and $G^{\prime \prime}$ change little with the increase in temperature and $G^{\prime \prime}$ is much larger than $G^{\prime}$, indicating that viscosity is dominant in this system at these temperatures. With further increasing temperature from $29^{\circ} \mathrm{C}, G^{\prime}$ and $G^{\prime \prime}$ began to increase. When temperature is around $33.5^{\circ} \mathrm{C}, G^{\prime}$ is close to $G^{\prime \prime}$ and stops increasing with temperature. This tempera-

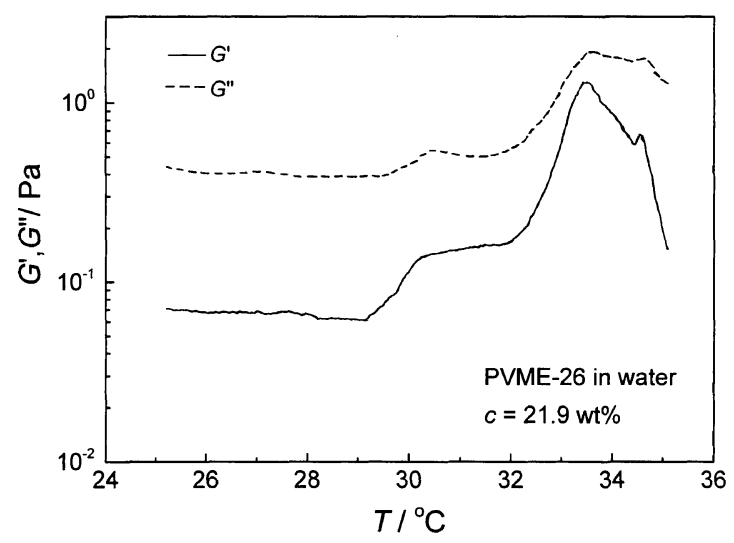

Figure 5. Change in dynamic moduli $G^{\prime}$ and $G^{\prime \prime}$ with increasing temperature measured at the angular frequency of $10 \mathrm{rad} \mathrm{s}^{-1}$ for PVME-26/water solution with the concentration of $21.9 \mathrm{wt} \%$.

ture dependence of $G^{\prime}$ and $G^{\prime \prime}$ manifests that the PVME/ water solution turns abruptly into a network structure in the vicinity of phase separation temperature. In other words, the gathering of PVME macromolecules to crosslink chains by hydrophobic bonding is the origin of the phase separation in the aqueous solution due to the appearance of suspended gel floc. This gelation process shows good reversibility and reproducibility as the solution is cooled down and heated again under the same condition. Therefore, what formed in PVME/water solution upon heating belongs to thermoreversible physical gel. ${ }^{1}$ The decrease in both $G^{\prime}$ and $G^{\prime \prime}$ with increasing temperature above $34^{\circ} \mathrm{C}$ may be attributed to the formation of floccular aggregation by the PVME network and weakening of the linkage among these flocculi at higher temperatures.

Acknowledgment. Financial support to this work by the National Natural Science Foundation (29725411) is gratefully acknowledged.

\section{REFERENCES}

1. K. te Nijenhuis, Thermoreversible Networks. Adv. Polym. Sci., 130, 1 (1997).

2. S. Callister, A. Keller, and R. M. Hikmet, Macromol. Chem., Macromol. Symp., 39, 19 (1990).

3. J. M. Yu, S. Blacher, F. Brouers, G. L'Homme, and R. Jerome, Macromolecules, 30, 4619 (1997).

4. F. Zeng, X. Zheng, and Z. Tong, Polymer, 39, 1249 (1998).

5. X. Zheng, Z. Tong, X. Xie, and F. Zeng, Polym. J., 30, 284 (1998).

6. F. Zeng, Z. Tong, and T. Sato, Sci. in China, Series B, 42, 290 (1999).

7. Z. Tong, F. Zeng, X. Zheng, and T. Sato, Macromolecules, 32 4488 (1999).

8. F. Zeng, X. Liu, Z. Tong, Y. Yang, and S. Wu, Sci. in China, Series $B, 43,428$ (2000).

9. H. G. Schild, Prog. Polym. Sci., 17, 163 (1992).

10. Y. Hirokawa and T. Tanaka, J. Chem. Phys., 81, 6379 (1984).

11. F. Zeng, Z. Tong, and H. Feng, Polymer, 38, 5539 (1997).

12. R. A. Horne, J. P. Almeida, A. F. Day, and N. T. Yu, J. Colloid Interface Sci., 35, 77 (1971).

13. H. G. Schild and D. A. Tirrell, J. Phys. Chem., 94, 4352 (1990).

14. J. A. Manson and G. J. Arquette, Macromol. Chem., 37, 18 (1960). 
15. D. Patterson, Macromolecules, 2, 672 (1969).

16. G. Nemethy and H. A. Scheraga, J. Chem. Phys., 36, 3382 (1962).

17. G. Nemethy and H. A. Scheraga, J. Chem. Phys., 36, 3401 (1962).
18. G. Nemethy and H. A. Scheraga, J. Phys. Chem., 66, 1773 (1962).

19. J. D. Ferry, "Viscoelasticity Properties of polymer", 3rd ed, John Wiley \& Sons, Inc., New York, N.Y., 1980. 